\title{
Paleoproterozoic glaciation: Evidence from carbon isotope record of the Hutuo Group, Wutai Mountain area of Shanxi Province, China
}

\author{
KONG FanFan ${ }^{1,2 *}$ YUAN XunLai ${ }^{2} \&$ ZHOU ChuanMing ${ }^{2}$ \\ ${ }^{1}$ School of Resource and Earth Science, China University of Mining and Technology, Xuzhou 221008, China; \\ ${ }^{2}$ State Key Laboratory of Paleobiology and Stratigraphy, Nanjing Institute of Geology and Paleontology, Chinese Academy of Sciences, Nanjing \\ 210008, China
}

Received January 20, 2011; accepted June 10, 2011

\begin{abstract}
Palaeoproterozoic global glaciation ( 2.3 Ga) may have been the first "snowball" Earth event in Earth history. North China has well-exposed sedimentary deposits of this time period with minimal postdepositional alteration. Sedimentary evidence of a glaciation event has been lacking, however. Here we analyzed carbon isotope composition of the Paleoproterozoic Hutuo Group (2.5$2.2 \mathrm{Ga}$ ) in the Wutai Mountain area, Shanxi Province, North China. Our data show, in an older to younger chronostratigraphic order, (1) positive $\delta^{13} \mathrm{C}_{\text {carb }}$ values in the Dashiling Formation of the Doucun Subgroup (3.2\%o to 1.0\%, VPDB); (2) a decrease in $\delta^{13} \mathrm{C}_{\text {carb }}$ values from the Wenshan Formation to the middle Daguandong Formation (from $2.0 \%$ to $-1.2 \%$ ), during which time the occurrence of stromatolites declined; (3) a pronounced negative excursion in the upper Daguandong Formation, at the boundary of the Daguandong and Huaiyincun formations (from 1.4\% to -3.3\%o), where stromatolites disappeared; and (4) a gradual increase of $\delta^{13} \mathrm{C}_{\text {carb }}$ values in the Beidaxing and Tianpengnao formations (from $-1.2 \%$ o to $1.4 \%$ ), during which time stromatolites returned. We argue that the negative carbon isotope excursion recorded from the Jian'ancun Formation to the middle Daguandong Formation of the Hutuo Group may have been North China's response to the Palaeoproterozoic global glaciation.
\end{abstract}

ice age, carbon isotopes, Hutuo Group, Paleoproterozoic, Wutai Mountain, Shanxi Province

Citation: Kong F F, Yuan X L, Zhou C M. Paleoproterozoic glaciation: Evidence from carbon isotope record of the Hutuo Group, Wutai Mountain area of Shanxi Province, China. Chinese Sci Bull, 2011, 56: 2922-2930, doi: 10.1007/s11434-011-4639-0

Stable isotope data have been used widely in reconstructing paleoenvironmental and paleoclimatic changes, especially in the early Earth, where fossil evidence is lacking. Much research progress has been made for this time period, including evaluation of the early Earth's atmosphere and ocean through studies of the changes in sulfur, carbon and oxygen isotopic compositions [1,2].

Paleoproterozoic $(\sim 2.3 \mathrm{Ga})$ low latitude glacial deposits are well preserved in South Africa (e.g. the Makganyene diamictite) [3], North America (e.g. the Huronian diamictite) [4], and Fennoscandia (Finland, Norway, Sweden and Denmark), suggesting a global glaciation event $[2,3]$. The cause of this "snowball" Earth event has been linked to the Great Oxidation event [5]. It is believed that the methane-rich

\footnotetext{
*Corresponding author (email: kongfanfan1981@sina.com)
}

Paleoproterozoic atmosphere may have kept the Earth surface temperature above the freezing point [6], and subsequent increasing in atmospheric oxygen may have reduced methane concentrations, ultimately triggering the Paleoproterozoic global glaciation $[4,7]$.

A Paleoproterozoic succession, spanning from 2.5 to 2.2 Ga, is well exposed in the Wutai Mountain area, Shanxi Province. No diamictite or any distinct glacial sedimentological feature has been reported in the succession, however. If the Paleoproterozoic glaciation was truly global, the $\mathrm{Wu}-$ tai Mountain area in North China should have recorded its signal. Carbon and oxygen isotope measurements have been carried out on Hutuo Group carbonates previously [8,9], interpretation of the data in the context of global glaciation is missing. To look for the stable isotope imprint of a global glaciation event in the Hutuo Group, we collected 205 
carbonate samples from the Hutuo Group and conducted carbon and oxygen isotopic analysis.

\section{Geological setting}

The Wutai Metamorphic Complex is located between the Fuping and Hengshan metamorphic complexes. Together they constitute the Hengshan-Wutai-Fuping orogen that makes up the middle segment of the Trans-North China Orogen. The Trans-North China Orogen is a narrow, northeast-southwest extending zone, which separates the North China Craton into eastern and western blocks (Figure 1).

The Wutai Metamorphic Complex is a greenstone terrane that consists of tonalitic-trondhjemitic-granodioritic gneisses, granitoids, mafic to felsic volcanic rocks, and metamorphosed volcanic-sedimentary rocks [10]. The Wutai granitegreenstone terrane is mainly composed of the Neoarchean Wutai Group and Paleoproterozoic Hutuo Group which comprise subgreenschist facies metasediments and minor mafic volcanics, including quartzites, slates, and metabasalts.
The Hutuo Group is considered the youngest unit in the Hengshan-Wutai-Fuping orogen. It unconformably overlies the Wutai Group, the latter of which interfinger with the Hutuo Group in some areas [10]. The Hutuo Group is distributed in an area of about $1500 \mathrm{~km}^{2}$, from northernmost Taihuai-Sijizhuang on the south slope of Wutai Mountain to southernmost Shizui-Dingxiang, and from the upper Taishan River to the east, to Yuanpingqi village in the west. The Hutuo Group also crops out from Daixian to Yuanping and Baishi on the northern slope of Wutai Mountain, as well as in Zhongtai in Fanshi [11].

The Hutuo Group is composed of metamorphic-conglomerate and quartzite at its base, dolostone and marble in central portions, and phyllite and slate interbedded with dolostone in its upper part, representing a complete sedimentary cycle [11]. Although the Hutuo Group succession has locally undergone intense folding and faulting during the younger Lüliang orogeny, many primary structures are well preserved, such as rhythmic bedding, ripple marks and cross bedding, as well as stromatolites in carbonate.

The Hutuo Group is subdivided into the Doucun, Dongye

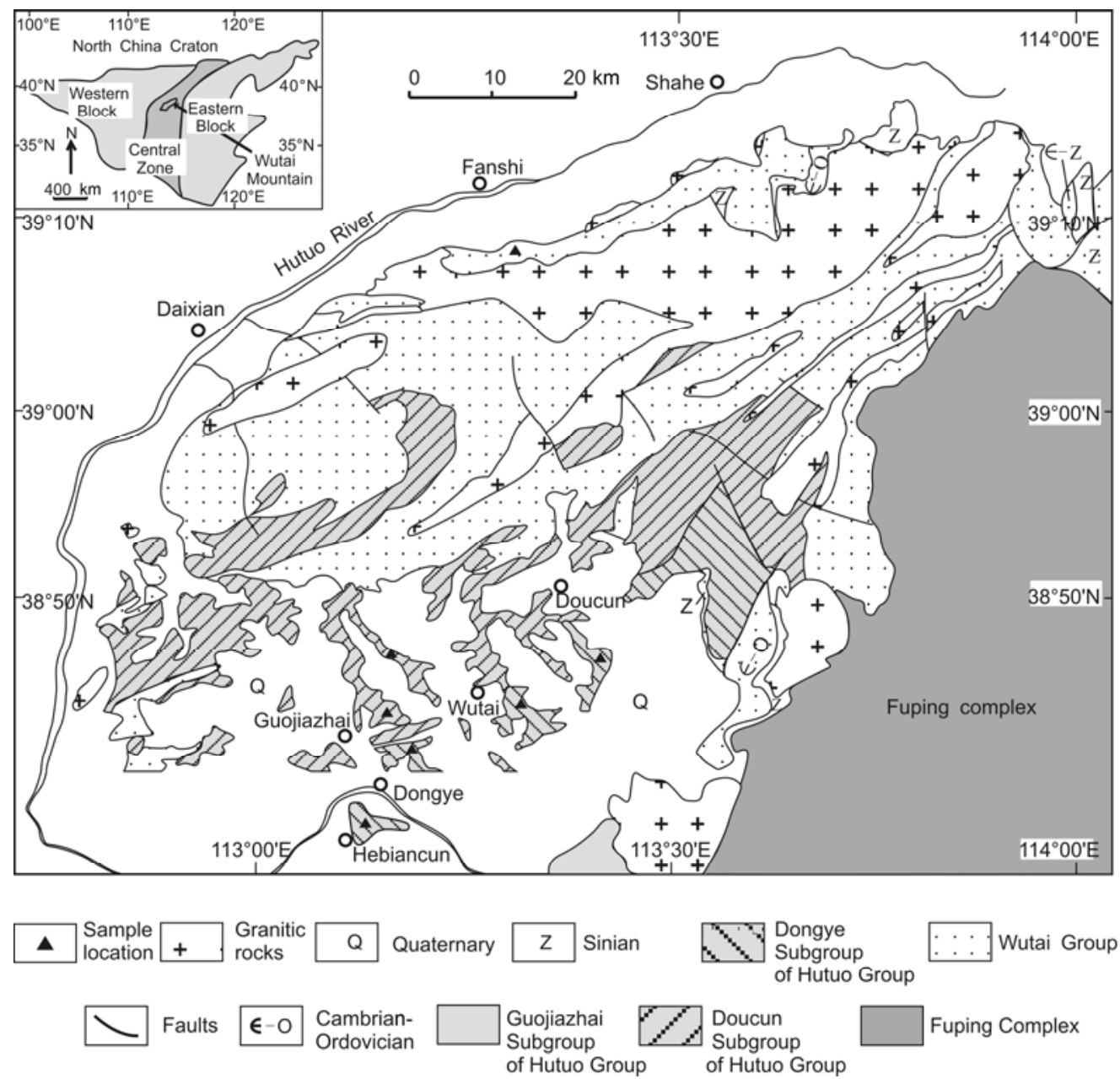

Figure 1 Geological map of the Wutai Mountain area (modified from Bai [11]). 
and Guojiazhai subgroups. The Doucun and Dongye subgroups are composed of 11 formations; in ascending order they are: Sijizhuang, Nantai, Dashiling, Qingshicun, Wenshan, Hebiancun, Jian'ancun, Daguandong, Huaiyincun, Beidaxing and Tianpengnao. The Sijizhuang Formation consists mainly of conglomerate, and minor metamorphosed sandstone and mudstone, representing fluvial deposits. The Nantai Formation is characterized by conglomerate, sandstone and limestone, indicting a transition from restricted lagoon to open shelf. The Dashiling Formation is mainly composed of sandstone, shale and limestone, reflecting environmental change from tidal flats to subtidal shallow water conditions. The Qingshicun Formation consists mainly of mudstone, with siltstone and dolomitic limestone interbeds, and basalt at the top of the formation. The Qingshicun Formation is inferred to have been deposited in shoreline or shore delta environments [11]. The Wenshan Formation constitutes sandstone overlain by shale and limestone; whereas, the Hebiancun Formation is characterized by sandstone and limestone, with basalt interbeds at the top. Both the Wenshan and Hebiancun formations were deposited in the tidal zone [11]. The Jian'ancun Foramation is mainly composed of thick-bedded dolomitic limestone, which was deposited in tidal zone environments. The Daguandong Formation consists of pink dolomitic limestone interbedded with grayish green slate, representing an open shallow marine environment. The Huaiyincun Formation is characterized by dolomitic limestone with a few stromatolites, indicating a low energy subtidal environment. The Beidaxing Formation is composed of thick-bedded, pink dolomitic limestone, which was deposited in a high energy shallow marine environment. The Tianpengnao Formation is characterized by interbeded, pink calcareous dolostone and shale, with lensoidal dolostone at the top, indicating an intertidal depositional setting.

A basic metavolcanite from the base of the Hutuo Group yields a zircon SHRIMP U-Pb age of $2517 \pm 13$ Ma. SHRIMP U-Pb zircon ages of $2529 \pm 10$ Ma and $2553 \pm 17$ Ma were obtained from two granitic gravels in the Sijizhuang Formation conglomerate. This age range is also confirmed with a SHRIMP zircon age of $2523 \mathrm{Ma}$ from arkose of the Sijizhuang Formation. Therefore, the maximum age of the Hutuo Group is conservatively about $2500 \mathrm{Ma}$ [12].

Meta-basalts from the Qingshicun Formation at the top of the Doucun Subgroup and those from the Hebiancun Formation at the base of the Dongye Subgroup are the best rocks for dating the Hutuo Group. Wu et al. [13] reported a conventional U-Pb zircon age of $2366 \pm 94$ Ma for Liudingsi basic metavocanics of the Qingshicun Formation. Wang et al. [14,15] reported SHRIMP U-Pb zircon ages of $2450 \pm 10$ $\mathrm{Ma}$ and $2400 \pm 20 \mathrm{Ma}$ for the basic metavolcanics of the Qingshicunand Hebiancun formations, respectively. Therefore, the boundary age of the Dongye Subgroup is about $2350 \mathrm{Ma}[12]$.

In 2003, Wilde et al. [16] reported SHRIMP U-Pb zircon ages of $2180 \pm 5 \mathrm{Ma}$ and $2087 \pm 9 \mathrm{Ma}$ for felsic tuffaceous rocks in the Tianpengnao Formation of Dongye Subgroup, near Taihuai in Wutai County. They suggested that the Hutuo Group should be older than $2180 \mathrm{Ma}$. Therefore, it can be inferred that the minimum age of the Dongye Subgroup is about $2.2 \mathrm{Ga}$ [12].

In summary, the Hutuo Group (excluding the Guojiazhai Subgroup) belongs to the early Paleoproterozoic (2.5 to 2.2 $\mathrm{Ga})$. The minimum age of the Doucun Subgroup is about $2350 \mathrm{Ma}$, and the minimum age of Dongye Subgroup is about $2200 \mathrm{Ma}$.

Columnar stromatolites occur in carbonate interbeds at the base of the phyllite of the Guquanshan Member, Dashiling Formation, which is considered as the lowermost stromatolite horizon of the Hutuo Group. Stromatolites are abundant in the Wenshan and Hebiancun formations, but decline rapidly in the Jian'ancun Formation, stay at low levels in the Daguandong Formation, and are absent in the Huaiyincun Formation [11]. Upward in the Beidaxing and Tianpengnao formations, stromatolites occur abundantly.

The oldest red beds-grayish red phyllite and slateappear in the Nantai Formation of the Doucun Subgroup. Red beds also occur in the Beidaxing Formation, and carbonates in the Hutuo Group are mainly red in color. Several meters of a basaltic paleo-weathering crust with clear zonation occurs at the top of the Qingshicun Formation. The $\mathrm{Fe}_{2} \mathrm{O}_{3} / \mathrm{FeO}$ ratios in ascending order rise from 0.4 to 15.48 [17]. The occurrence of red beds, stromatolites and weathering crusts suggest that sediments were becoming oxidized during that time period.

\section{Methods}

We collected samples of micritic limestone, dolomitic limestone, calcareous dolostone and a small quantity of marble from the Doucun and Dongye subgroups at several sections (Table 1). The carbonate-dominated sections were measured and samples of fresh microcrystalline limestone or microcrystalline dolostone without late veins were collected with a sampling interval of 5 to $8 \mathrm{~m}$. In this study, carbon and oxygen isotopic measurements were conducted for 205 samples. $\mathrm{CO}_{2}$ was extracted using standard offline techniques, where samples were reacted with concentrated $\mathrm{H}_{3} \mathrm{PO}_{4}$ at $50^{\circ} \mathrm{C}$ for $12 \mathrm{~h} \mathrm{[18].} \mathrm{Carbon} \mathrm{and} \mathrm{oxygen} \mathrm{isotopic}$ ratios were determined on a Finnigan MAT 253 mass spectrometer at Nanjing Institute of Geology and Palaeontology, Chinese Academy of Sciences. Analytical precision is better than $0.1 \%$ for $\delta^{13} \mathrm{C}$ and $0.1 \%$ for $\delta^{18} \mathrm{O}$. Isotopic data are reported in \%o VPDB (Table 2).

\section{Results and data evaluation}

The carbon isotopic composition of marine carbonate can 
Table 1 Sampling horizon and profile position of the Paleoproterozoic Hutuo Group

\begin{tabular}{|c|c|c|c|}
\hline \multicolumn{3}{|c|}{ Sampling horizon } & \multirow{2}{*}{ Profile position } \\
\hline Group & Subgroup & Formation & \\
\hline \multirow[t]{10}{*}{ Hutuo } & Dongye & Tianpengnao & Geziling-Luojianao section east of Wutai County \\
\hline & & Beidaxing & Luojianao section, southeast of Wutai \\
\hline & & Huaiyincun & Ma'anshan section near Huaiyin village in Wutai County \\
\hline & & The top of Daguandong & \\
\hline & & Daguandong (excluding the top of it) & Wenshan-Daguanshan section in Dingxiang and Wutai Counties \\
\hline & & Jian'ancun & \\
\hline & & Hebiancun & \\
\hline & & Wenshan & \\
\hline & Doucun & Nandaxian Member & Xilangshan section in Wutai County \\
\hline & & Pandaoling Member & Guquanshan-Shenxiannao section in Wutai County \\
\hline Wutai & Taihuai & Baizhiyan & Baizhiyancun section near Taipinggou village, Fanshi County \\
\hline
\end{tabular}

Table $2 \delta^{13} \mathrm{C}$ and $\delta^{18} \mathrm{O}$ data of the Paleoproterozoic Hutuo Group

\begin{tabular}{|c|c|c|c|c|c|c|c|}
\hline Number & $\delta^{13} \mathrm{C}(\%$, VPDB $)$ & $\delta^{18} \mathrm{O}(\%, \mathrm{VPDB})$ & Thickness (m) & Number & $\delta^{13} \mathrm{C}(\%, \mathrm{VPDB})$ & $\delta^{18} \mathrm{O}(\%, \mathrm{VPDB})$ & Thickness (m) \\
\hline $\mathrm{H}^{\mathrm{tpn}}-27$ & 1.0 & -12.4 & 12979 & $\mathrm{H}^{\mathrm{bdx}}-17$ & -1.2 & -8.7 & 11446 \\
\hline $\mathrm{H}^{\mathrm{tpn}}-26$ & 0.8 & -12.3 & 12976 & $\mathrm{H}^{\mathrm{bdx}}-16$ & -0.9 & -6.1 & 11444 \\
\hline $\mathrm{H}^{\mathrm{tpn}}-25$ & 1.4 & -11.9 & 12961 & $\mathrm{H}^{\mathrm{bdx}}-15$ & -0.3 & -7.2 & 11436 \\
\hline $\mathrm{H}^{\mathrm{tpn}}-24$ & 1.2 & -11.1 & 12944 & $\mathrm{H}^{\mathrm{bdx}}-14$ & -0.3 & -6.3 & 11425 \\
\hline $\mathrm{H}^{\mathrm{tpn}}-23$ & 1.2 & -10.8 & 12941 & $\mathrm{H}^{\mathrm{bdx}}-13$ & -0.3 & -7.1 & 11415 \\
\hline $\mathrm{H}^{\mathrm{tpn}}-22$ & 1.0 & -10.6 & 12936 & $\mathrm{H}^{\mathrm{bdx}}-12$ & -0.7 & -8.0 & 11406 \\
\hline $\mathrm{H}^{\mathrm{tpn}}-21$ & 0.9 & -10.7 & 12933 & $\mathrm{H}^{\mathrm{bdx}}-11$ & -0.4 & -13.3 & 11399 \\
\hline $\mathrm{H}^{\mathrm{tpn}}-20$ & 0.9 & -10.7 & 12928 & $\mathrm{H}^{\mathrm{bdx}}-10$ & -0.0 & -6.7 & 11374 \\
\hline $\mathrm{H}^{\mathrm{tpn}}-19$ & 0.4 & -10.8 & 12921 & $\mathrm{H}^{\mathrm{bdx}}-9$ & -0.0 & -6.4 & 11367 \\
\hline $\mathrm{H}^{\mathrm{tpn}}-18$ & 0.4 & -10.7 & 12911 & $\mathrm{H}^{\mathrm{bdx}}-8$ & 0.1 & -7.1 & 11363 \\
\hline $\mathrm{H}^{\mathrm{tpn}}-17$ & 0.1 & -10.5 & 12901 & $\mathrm{H}^{\mathrm{bdx}}-7$ & 0.0 & -7.1 & 11357 \\
\hline $\mathrm{H}^{\mathrm{tpn}}-16$ & 0.7 & -10.8 & 12889 & $\mathrm{H}^{\mathrm{bdx}}-6$ & -0.3 & -7.1 & 11356 \\
\hline $\mathrm{H}^{\mathrm{tpn}}-15$ & 0.5 & -10.6 & 12871 & $\mathrm{H}^{\mathrm{bdx}}-5$ & -0.4 & -7.1 & 11352 \\
\hline $\mathrm{H}^{\mathrm{tpn}}-14$ & 1.1 & -10.6 & 12861 & $\mathrm{H}^{\mathrm{bdx}}-4$ & -0.4 & -6.5 & 11316 \\
\hline $\mathrm{H}^{\mathrm{tpn}}-13$ & 1.0 & -10.4 & 12846 & $\mathrm{H}^{\mathrm{bdx}}-3$ & -0.3 & -7.4 & 11308 \\
\hline $\mathrm{H}^{\mathrm{tpn}}-12$ & 1.2 & -10.3 & 12839 & $\mathrm{H}^{\mathrm{bdx}}-2$ & -0.3 & -7.2 & 11298 \\
\hline $\mathrm{H}^{\mathrm{tpn}}-11$ & 1.2 & -10.5 & 12835 & $\mathrm{H}^{\mathrm{bdx}}-1$ & -0.2 & -7.1 & 11275 \\
\hline $\mathrm{H}^{\mathrm{tpn}}-10$ & 1.1 & -10.4 & 12834 & $\mathrm{H}^{\mathrm{hyc}}-21$ & -2.5 & -8.9 & 10551 \\
\hline $\mathrm{H}^{\mathrm{tpn}}-9$ & 1.2 & -10.4 & 12829 & $\mathrm{H}^{\mathrm{hyc}}-20$ & -2.5 & -8.9 & 10548 \\
\hline $\mathrm{H}^{\mathrm{tpn}}-8$ & 1.1 & -10.6 & 12777 & $\mathrm{H}^{\mathrm{hyc}}-19$ & -2.4 & -8.2 & 10539 \\
\hline $\mathrm{H}^{\mathrm{tpn}}-7$ & 0.3 & -10.8 & 12772 & $\mathrm{H}^{\mathrm{hyc}}-18$ & -2.3 & -8.2 & 10527 \\
\hline $\mathrm{H}^{\mathrm{tpn}}-6$ & 0.0 & -11.3 & 12767 & $\mathrm{H}^{\text {hyc }}-17$ & -2.0 & -8.7 & 10510 \\
\hline $\mathrm{H}^{\mathrm{tpn}}-5$ & 0.1 & -11.7 & 12760 & $\mathrm{H}^{\mathrm{hyc}}-16$ & -2.0 & -10.4 & 10499 \\
\hline $\mathrm{H}^{\mathrm{tpn}}-4$ & 1.0 & -9.7 & 12742 & $\mathrm{H}^{\mathrm{hyc}}-15$ & -0.8 & -5.9 & 10484 \\
\hline $\mathrm{H}^{\mathrm{tpn}}-3$ & 0.8 & -9.7 & 12733 & $\mathrm{H}^{\mathrm{hyc}}-14$ & -0.7 & -7.7 & 10477 \\
\hline $\mathrm{H}^{\mathrm{tpn}}-2$ & -0.9 & -9.3 & 12694 & $\mathrm{H}^{\text {hyc }}-13$ & -0.8 & -5.9 & 10460 \\
\hline $\mathrm{H}^{\mathrm{tpn}}-1$ & -0.6 & -11.4 & 12688 & $\mathrm{H}^{\mathrm{hyc}}-12$ & -1.2 & -7.6 & 10448 \\
\hline $\mathrm{H}^{\mathrm{bdx}}-30$ & -0.9 & -8.0 & 12464 & $\mathrm{H}^{\mathrm{hyc}}-11$ & -0.8 & -6.5 & 10433 \\
\hline $\mathrm{H}^{\mathrm{bdx}}-29$ & -0.7 & -7.6 & 12461 & $\mathrm{H}^{\mathrm{hyc}}-10$ & -1.6 & -6.1 & 10424 \\
\hline $\mathrm{H}^{\mathrm{bdx}}-28$ & -0.6 & -6.4 & 12458 & $\mathrm{H}^{\mathrm{hyc}} \_9$ & -1.7 & -9.3 & 10416 \\
\hline $\mathrm{H}^{\mathrm{bdx}}-27$ & -0.6 & -6.4 & 12455 & $\mathrm{H}^{\text {hyc }}-8$ & -2.0 & -7.9 & 10404 \\
\hline
\end{tabular}


(Continued)

\begin{tabular}{|c|c|c|c|c|c|c|c|}
\hline Number & $\delta^{13} \mathrm{C}(\%, \mathrm{VPDB})$ & $\delta^{18} \mathrm{O}(\%, \mathrm{VPDB})$ & Thickness (m) & Number & $\delta^{13} \mathrm{C}(\%, \mathrm{VPDB})$ & $\delta^{18} \mathrm{O}(\% o, \mathrm{VPDB})$ & Thickness (m) \\
\hline $\mathrm{H}^{\mathrm{bdx}}-26$ & -0.5 & -6.9 & 12452 & $\mathrm{H}^{\text {hyc }}-7$ & -2.0 & -8.2 & 10396 \\
\hline $\mathrm{H}^{\mathrm{bdx}}-25$ & -0.3 & -7.0 & 12449 & $\mathrm{H}^{\text {hyc }}-6$ & -2.2 & -8.8 & 10371 \\
\hline $\mathrm{H}^{\mathrm{bdx}}-24$ & -0.3 & -6.0 & 12446 & $\mathrm{H}^{\text {hyc }}-5$ & -3.3 & -9.4 & 10347 \\
\hline $\mathrm{H}^{\mathrm{bdx}}-23$ & -0.4 & -6.3 & 11486 & $\mathrm{H}^{\text {hyc }}-4$ & -3.2 & -9.4 & 10345 \\
\hline $\mathrm{H}^{\mathrm{bdx}}-22$ & -0.9 & -7.1 & 11478 & $\mathrm{H}^{\text {hyc }}-3$ & -3.3 & -10.2 & 10342 \\
\hline $\mathrm{H}^{\mathrm{bdx}}-21$ & -0.7 & -6.5 & 11470 & $\mathrm{H}^{\text {hyc }}-2$ & -3.1 & -9.7 & 10335 \\
\hline $\mathrm{H}^{\mathrm{bdx}}-20$ & -0.8 & -6.7 & 11466 & $\mathrm{H}^{\text {hyc }}-1$ & -2.1 & -9.3 & 10327 \\
\hline $\mathrm{H}^{\mathrm{bdx}}-19$ & -0.2 & -6.3 & 11456 & $\mathrm{H}^{\mathrm{dgd}}-53$ & -0.2 & -6.9 & 10318 \\
\hline $\mathrm{H}^{\mathrm{bdx}}-18$ & -1.1 & -6.7 & 11451 & $\mathrm{H}^{\mathrm{dgd}}-52$ & 1.3 & -9.5 & 10307 \\
\hline $\mathrm{H}^{\mathrm{dgd}}-51$ & 1.2 & -9.8 & 10291 & $\mathrm{H}^{\mathrm{dgd}}-9$ & -0.8 & -7.6 & 9367 \\
\hline $\mathrm{H}^{\mathrm{dgd}}-50$ & 0.9 & -9.7 & 10265 & $\mathrm{H}^{\mathrm{dgd}}-8$ & -0.9 & -7.8 & 9358 \\
\hline $\mathrm{H}^{\mathrm{dgd}}-49$ & -2.7 & -6.6 & 10252 & $\mathrm{H}^{\mathrm{dgd}}-7$ & -0.9 & -7.8 & 9295 \\
\hline $\mathrm{H}^{\mathrm{dgd}}-48$ & -2.0 & -7.0 & 10246 & $\mathrm{H}^{\mathrm{dgd}}-6$ & -0.2 & -9.1 & 9277 \\
\hline $\mathrm{H}^{\mathrm{dgd}}-47$ & -3.3 & -10.5 & 10220 & $\mathrm{H}^{\mathrm{dgd}}-5$ & -0.2 & -6.6 & 9266 \\
\hline $\mathrm{H}^{\mathrm{dgd}}-46$ & -3.3 & -7.1 & 10208 & $\mathrm{H}^{\mathrm{dgd}}-4$ & -0.3 & -7.5 & 9252 \\
\hline $\mathrm{H}^{\mathrm{dgd}}-45$ & 0.4 & -11.3 & 10189 & $\mathrm{H}^{\mathrm{dgd}}-3$ & 0.9 & -10.2 & 9247 \\
\hline $\mathrm{H}^{\mathrm{dgd}}-44$ & 0.2 & -11.0 & 10173 & $\mathrm{H}^{\mathrm{dgd}}-2$ & 0.6 & -9.6 & 9235 \\
\hline $\mathrm{H}^{\mathrm{dgd}}-43$ & -1.2 & -13.2 & 10152 & $\mathrm{H}^{\mathrm{dgd}}-1$ & -0.1 & -7.7 & 9228 \\
\hline $\mathrm{H}^{\mathrm{dgd}}-42$ & 0.4 & -10.6 & 10139 & $\mathrm{H}^{\mathrm{jac}}-28$ & -0.9 & -9.3 & 8148 \\
\hline $\mathrm{H}^{\mathrm{dgd}}-41$ & 0.5 & -10.2 & 10138 & $\mathrm{H}^{\mathrm{jac}}-27$ & -1.3 & -7.7 & 8145 \\
\hline $\mathrm{H}^{\mathrm{dgd}}-40$ & 1.4 & -9.8 & 10124 & $\mathrm{H}^{\mathrm{jac}}-26$ & -1.2 & -8.4 & 8134 \\
\hline $\mathrm{H}^{\mathrm{dgd}}-39$ & 0.3 & -9.8 & 10118 & $\mathrm{H}^{\mathrm{jac}}-25$ & 1.1 & -7.9 & 8122 \\
\hline $\mathrm{H}^{\mathrm{dgd}}-38$ & 0.1 & -9.8 & 10106 & $\mathrm{H}^{\mathrm{j} a \mathrm{c}}-24$ & -0.5 & -7.5 & 8115 \\
\hline $\mathrm{H}^{\mathrm{dgd}}-37$ & 0.3 & -10.2 & 10097 & $\mathrm{H}^{\mathrm{jac}}-23$ & -0.5 & -6.6 & 8114 \\
\hline $\mathrm{H}^{\mathrm{dgd}}-36$ & 0.2 & -10.6 & 10084 & $\mathrm{H}^{\mathrm{jac}}-22$ & -0.4 & -6.7 & 8111 \\
\hline $\mathrm{H}^{\mathrm{dgd}}-35$ & 0.2 & -10.6 & 10070 & $\mathrm{H}^{\mathrm{jac}}-21$ & -0.6 & -6.6 & 8105 \\
\hline $\mathrm{H}^{\mathrm{dgd}}-34$ & 0.2 & -11.2 & 10061 & $\mathrm{H}^{\mathrm{jac}}-20$ & -0.4 & -6.2 & 8101 \\
\hline $\mathrm{H}^{\mathrm{dgd}}-33$ & 0.1 & -11.1 & 10055 & $\mathrm{H}^{\mathrm{jac}}-19$ & -0.8 & -8.1 & 8097 \\
\hline $\mathrm{H}^{\mathrm{dgd}}-32$ & -0.3 & -9.1 & 10046 & $\mathrm{H}^{\mathrm{jac}}-18$ & 0.2 & -8.1 & 8095 \\
\hline $\mathrm{H}^{\mathrm{dgd}}-31$ & -0.2 & -7.1 & 10029 & $\mathrm{H}^{\mathrm{jac}}-17$ & 0.4 & -7.2 & 8094 \\
\hline $\mathrm{H}^{\mathrm{dgd}}-30$ & -0.1 & -6.9 & 10025 & $\mathrm{H}^{\mathrm{jac}}-16$ & 0.4 & -7.3 & 8093 \\
\hline $\mathrm{H}^{\mathrm{dgd}}-29$ & 1.4 & -10.0 & 10018 & $\mathrm{H}^{\mathrm{jac}}-15$ & 0.8 & -7.2 & 8091 \\
\hline $\mathrm{H}^{\mathrm{dgd}}-28$ & 0.8 & -9.3 & 10014 & $\mathrm{H}^{\mathrm{j} a \mathrm{c}}-14$ & 0.0 & -6.4 & 8089 \\
\hline $\mathrm{H}^{\mathrm{dgd}}-27$ & 0.4 & -9.9 & 9741 & $\mathrm{H}^{\mathrm{jac}}-13$ & 1.1 & -7.7 & 8086 \\
\hline $\mathrm{H}^{\mathrm{dgd}}-26$ & 1.5 & -7.4 & 9724 & $\mathrm{H}^{\mathrm{jac}}-12$ & 0.1 & -6.8 & 8078 \\
\hline $\mathrm{H}^{\mathrm{dgd}}-25$ & 0.0 & -6.6 & 9708 & $\mathrm{H}^{\mathrm{jac}}-11$ & 0.1 & -9.9 & 8075 \\
\hline $\mathrm{H}^{\mathrm{dgd}}-24$ & 1.1 & -8.7 & 9700 & $\mathrm{H}^{\mathrm{jac}}-10$ & 0.2 & -10.7 & 8072 \\
\hline $\mathrm{H}^{\mathrm{dgd}}-23$ & 1.1 & -7.2 & 9692 & $\mathrm{H}^{\mathrm{jac}}-9$ & 0.0 & -10.3 & 8055 \\
\hline $\mathrm{H}^{\mathrm{dgd}}-22$ & 1.0 & -8.3 & 9685 & $\mathrm{H}^{\mathrm{jac}}-8$ & 0.0 & -10.0 & 8052 \\
\hline $\mathrm{H}^{\mathrm{dgd}}-21$ & 1.0 & -8.4 & 9672 & $\mathrm{H}^{\mathrm{jac}}-7$ & 0.0 & -9.7 & 8051 \\
\hline $\mathrm{H}^{\mathrm{dgd}}-20$ & 1.1 & -8.4 & 9660 & $\mathrm{H}^{\mathrm{jac}}-6$ & 0.0 & -10.4 & 8040 \\
\hline $\mathrm{H}^{\mathrm{dgd}}-19$ & 1.2 & -8.0 & 9649 & $\mathrm{H}^{\mathrm{jac}}-5$ & 0.1 & -10.2 & 8039 \\
\hline $\mathrm{H}^{\mathrm{dgd}}-18$ & 0.5 & -8.0 & 9460 & $\mathrm{H}^{\mathrm{jac}}-4$ & 0.4 & -8.0 & 8035 \\
\hline $\mathrm{H}^{\mathrm{dgd}}-17$ & 0.2 & -6.8 & 9454 & $\mathrm{H}^{\mathrm{jac}}-3$ & 0.4 & -8.1 & 8030 \\
\hline $\mathrm{H}^{\mathrm{dgd}}-16$ & 0.4 & -7.8 & 9442 & $\mathrm{H}^{\mathrm{jac}}-2$ & 0.4 & -7.4 & 8025 \\
\hline $\mathrm{H}^{\mathrm{dgd}}-15$ & 0.3 & -9.5 & 9433 & $\mathrm{H}^{\mathrm{jac}}-1$ & 0.2 & -10.1 & 8020 \\
\hline
\end{tabular}


(Continued)

\begin{tabular}{|c|c|c|c|c|c|c|c|}
\hline Number & $\delta^{13} \mathrm{C}(\%, \mathrm{VPDB})$ & $\delta^{18} \mathrm{O}(\%, \mathrm{VPDB})$ & Thickness (m) & Number & $\delta^{13} \mathrm{C}(\%$, VPDB $)$ & $\delta^{18} \mathrm{O}(\%, \mathrm{VPDB})$ & Thickness (m) \\
\hline $\mathrm{H}^{\mathrm{dgd}}-14$ & -0.5 & -7.4 & 9426 & $\mathrm{H}^{\mathrm{ndx}}-7$ & 2.8 & -7.1 & 5771 \\
\hline $\mathrm{H}^{\mathrm{dgd}}-13$ & 0.4 & -7.7 & 9410 & $\mathrm{H}^{\mathrm{ndx}}-6$ & 2.5 & -6.7 & 5762 \\
\hline $\mathrm{H}^{\mathrm{dgd}}-12$ & -0.9 & -7.4 & 9392 & $\mathrm{H}^{\mathrm{ndx}}-5$ & 2.5 & -6.9 & 5753 \\
\hline $\mathrm{H}^{\mathrm{dgd}}-11$ & -0.8 & -8.1 & 9384 & $\mathrm{H}^{\mathrm{ndx}}-4$ & 1.7 & -8.2 & 5741 \\
\hline $\mathrm{H}^{\mathrm{dgd}}-10$ & -0.9 & -8.1 & 9373 & $\mathrm{H}^{\mathrm{ndx}}-3$ & 1.5 & -8.9 & 5721 \\
\hline $\mathrm{H}^{\mathrm{hbc}}-1$ & -0.2 & -6.9 & 7813 & $\mathrm{H}^{\mathrm{ndx}}-2$ & 2.0 & -9.7 & 5709 \\
\hline $\mathrm{H}^{\mathrm{ws}}-7$ & 0.1 & -9.3 & 7365 & $\mathrm{H}^{\mathrm{ndx}}-1$ & 1.0 & -10.7 & 5708 \\
\hline $\mathrm{H}^{\mathrm{ws}}-6$ & 0.3 & -9.2 & 7356 & $\mathrm{H}^{\mathrm{pdl}}-5$ & 2.2 & -13.0 & 5016 \\
\hline $\mathrm{H}^{\mathrm{ws}}-5$ & 1.1 & -9.2 & 7344 & $\mathrm{H}^{\mathrm{pdl}}-4$ & 1.3 & -13.3 & 4957 \\
\hline $\mathrm{H}^{\mathrm{ws}}-4$ & 1.4 & -10.7 & 7336 & $\mathrm{H}^{\mathrm{pdl}}-3$ & 1.8 & -13.1 & 4954 \\
\hline $\mathrm{H}^{\mathrm{ws}}-3$ & 0.7 & -9.8 & 7320 & $\mathrm{H}^{\mathrm{pdl}}-2$ & 2.9 & -13.0 & 4876 \\
\hline $\mathrm{H}^{\mathrm{ws}}-2$ & 1.1 & -9.7 & 7307 & $\mathrm{H}^{\mathrm{pdl}}-1$ & 3.2 & -13.0 & 4873 \\
\hline $\mathrm{H}^{\mathrm{ws}}-1$ & 2.0 & -9.5 & 7292 & $\mathrm{~W}^{\mathrm{bzy}}-8$ & 0.9 & -19.0 & 559 \\
\hline $\mathrm{H}^{\mathrm{ndx}}-20$ & 2.0 & -8.9 & 5848 & $\mathrm{~W}^{\mathrm{bzy}}-7$ & -0.9 & -18.4 & 542 \\
\hline $\mathrm{H}^{\mathrm{ndx}}-19$ & 2.4 & -7.7 & 5840 & $\mathrm{~W}^{\mathrm{bzy}}-6$ & 0.3 & -24.0 & 541 \\
\hline $\mathrm{H}^{\mathrm{ndx}}-18$ & 2.0 & -8.9 & 5835 & $\mathrm{~W}^{\mathrm{bzy}}-5$ & -2.7 & -22.0 & 514 \\
\hline $\mathrm{H}^{\mathrm{ndx}}-17$ & 2.7 & -7.6 & 5827 & $\mathrm{~W}^{\mathrm{bzy}}-4$ & -2.8 & -20.2 & 463 \\
\hline $\mathrm{H}^{\mathrm{ndx}}-16$ & 2.5 & -7.1 & 5821 & $\mathrm{~W}^{\mathrm{bzy}}-3$ & -3.3 & -21.2 & 328 \\
\hline $\mathrm{H}^{\mathrm{ndx}}-15$ & 2.6 & -7.4 & 5819 & $\mathrm{~W}^{\mathrm{bzy}}-2$ & -2.8 & -19.8 & 325 \\
\hline $\mathrm{H}^{\mathrm{ndx}}-14$ & 2.3 & -8.4 & 5811 & $\mathrm{H}^{\mathrm{hbc}}-6$ & 1.0 & -9.2 & 7955 \\
\hline $\mathrm{H}^{\mathrm{ndx}}-13$ & 2.6 & -6.9 & 5805 & $\mathrm{H}^{\mathrm{hbc}}-5$ & 1.7 & -7.8 & 7928 \\
\hline $\mathrm{H}^{\mathrm{ndx}}-12$ & 2.6 & -6.4 & 5798 & $\mathrm{H}^{\mathrm{hbc}}-4$ & 0.1 & -9.4 & 7909 \\
\hline $\mathrm{H}^{\mathrm{ndx}}-11$ & 2.4 & -7.2 & 5794 & $\mathrm{H}^{\mathrm{hbc}}-3$ & 0.8 & -8.1 & 7885 \\
\hline $\mathrm{H}^{\mathrm{ndx}}-10$ & 2.7 & -5.6 & 5789 & $\mathrm{H}^{\mathrm{hbc}}-2$ & 0.9 & -6.6 & 7859 \\
\hline $\mathrm{H}^{\mathrm{ndx}}-9$ & 2.4 & -8.8 & 5785 & $\mathrm{~W}^{\mathrm{bzy}}-1$ & -4.1 & -28.7 & 306 \\
\hline $\mathrm{H}^{\mathrm{ndx}}-8$ & 2.9 & -6.6 & 5779 & & & & \\
\hline
\end{tabular}

undergo alteration during post-depositional processes, including early diagenesis, late diagenetic rock-fluid exchange, hydrothermal activities, and metamorphism. The extent of carbon isotopic post-depositional changes [19] may be estimated by evaluating degree of crystallization, with finer grained microcrystalline limestone indicating minimal alteration. Similarly, weathered samples with later veins or diagenetic cementation are not suitable for isotopic measurement. Furthermore, samples with $\delta^{18} \mathrm{O}$ values $<-10 \%$ o have likely experienced considerable diagenetic alteration. Samples with no correlation between $\delta^{18} \mathrm{O}$ and $\delta^{13} \mathrm{C}$ values probably did not experience significant diagenetic alteration. The samples utilized in this study were fresh microcrystalline limestone or microcrystalline dolostone without later veins. Most samples yielded $\delta^{18} \mathrm{O}$ values more positive than $-10 \%$ (Table 2) and showed no apparent correlation between $\delta^{13} \mathrm{C}$ and $\delta^{18} \mathrm{O}$ values (Figure 2), suggesting that $\delta^{13} \mathrm{C}$ values may not have undergone significant post-

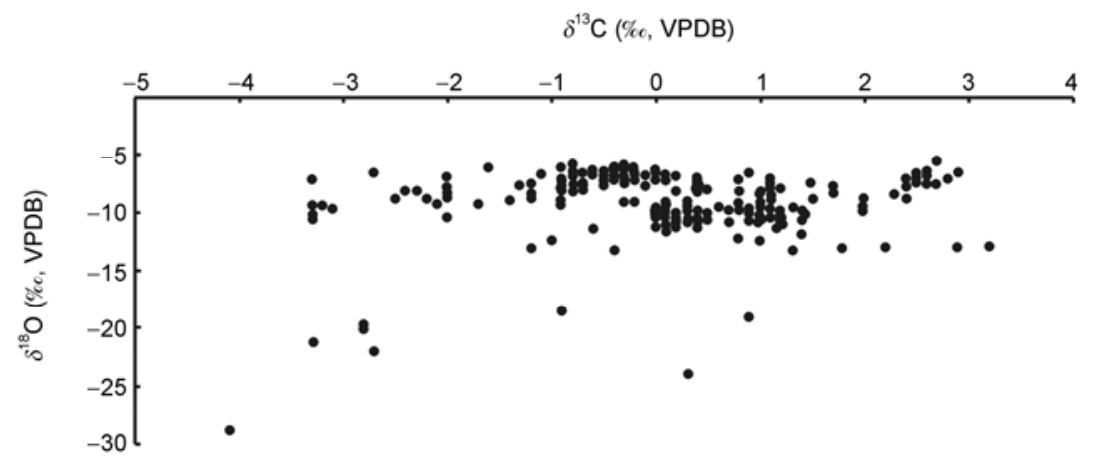

Figure $2 \delta^{13} \mathrm{C}-\delta^{18} \mathrm{O}$ cross-plot of carbonate samples of the Paleoproterozoic Hutuo Group. 
depositional alteration.

The variation of carbon isotope composition in the Paleoproterozoic Hutuo Group (2.5 to $2.2 \mathrm{Ga}$ ) can be divided into four parts from older to younger ages (Figure 3): (1) positive $\delta^{13} \mathrm{C}_{\text {carb }}$ values in the Dashiling Formation carbonates of the Doucun Subgroup (3.2\%o-1.0\%o, VPDB); (2) a decrease in $\delta^{13} \mathrm{C}_{\text {carb }}$ values from the Wenshan Formation to the middle of the Daguandong Formation (from 2.0\% to $-1.2 \%$ ); (3) a pronounced negative $\delta^{13} \mathrm{C}$ excursion in the upper Daguandong Formation and at the boundary of the Daguandong and Huaiyincun formations (from 1.4\%o to $-3.3 \%$ ); and (4) a gradual increase of $\delta^{13} \mathrm{C}_{\text {carb }}$ values in the Beidaxing and Tianpengnao formations (from $-1.2 \%$ to $1.4 \%$ ).

\section{Discussion and conclusions}

Both the Paleoproterozoic and Neoproterozoic witnessed the assembly and breakup of supercontinents [5], as well as extensive glaciations [4], which were associated with extreme carbon isotope variations [20]. Before glaciation, the marine carbonates have been shown to be enriched in ${ }^{13} \mathrm{C}$, and after glaciation, depleted ${ }^{13} \mathrm{C}$ [20]. A large numbers of work on Neoproterozoic glaciation may serve as an analog for the study of the Paleoproterozoic one.

Changes of carbon isotope compositions of marine carbonate and paleoclimate are believed to have been associated with plate tectonic and mantle plume events [21]. A Paleoproterozoic superplume was active between 2.51 to 2.45 $\mathrm{Ga}$ [5], during which time the upper mantle and lithosphere were heated up from beneath. Many basaltic eruptions produced oceanic plateaus, and thereby elevated sea level [21]. Volcanic eruptions and associated extensive hydrothermal activity released both $\mathrm{CO}_{2}$ and reductive gases into the atmosphere-ocean system. The greenhouse effect of increased atmospheric $\mathrm{CO}_{2}$ thus intensified continental weathering rates. Enhanced weathering and hydrothermal activity brought a large amount of nutrients into the ocean, which combined with the warm ocean water, evidently increased the ocean productivity initially. In general, organic matter burial is enhanced by sea-level rise and the expansion of anoxic waters [21]. Thus it can be seen that the Paleoproterozoic superplume event may have promoted the formation and deposition of organic carbon. An increase in burial rate of organic matter thus resulted in positive carbon isotope values in pre-glacial carbonates, corresponding to the high positive carbon isotope value in the Dashiling Formation ( $\delta^{13} \mathrm{C}_{\text {carb }}$ values range from $3.2 \%$ to $1.0 \%$ ) (Figure 3 ).

Concentration of atmospheric $\mathrm{O}_{2}$ increased and probably reached $\sim 10^{-5}$ PAL (present atmosphere level) at around $2.4 \mathrm{Ga}$ [5]. The rise of atmospheric oxygen decreased atmospheric methane concentration [4]. Meanwhile, increasing fixation of atmospheric $\mathrm{CO}_{2}$ into rock (carbonates) via

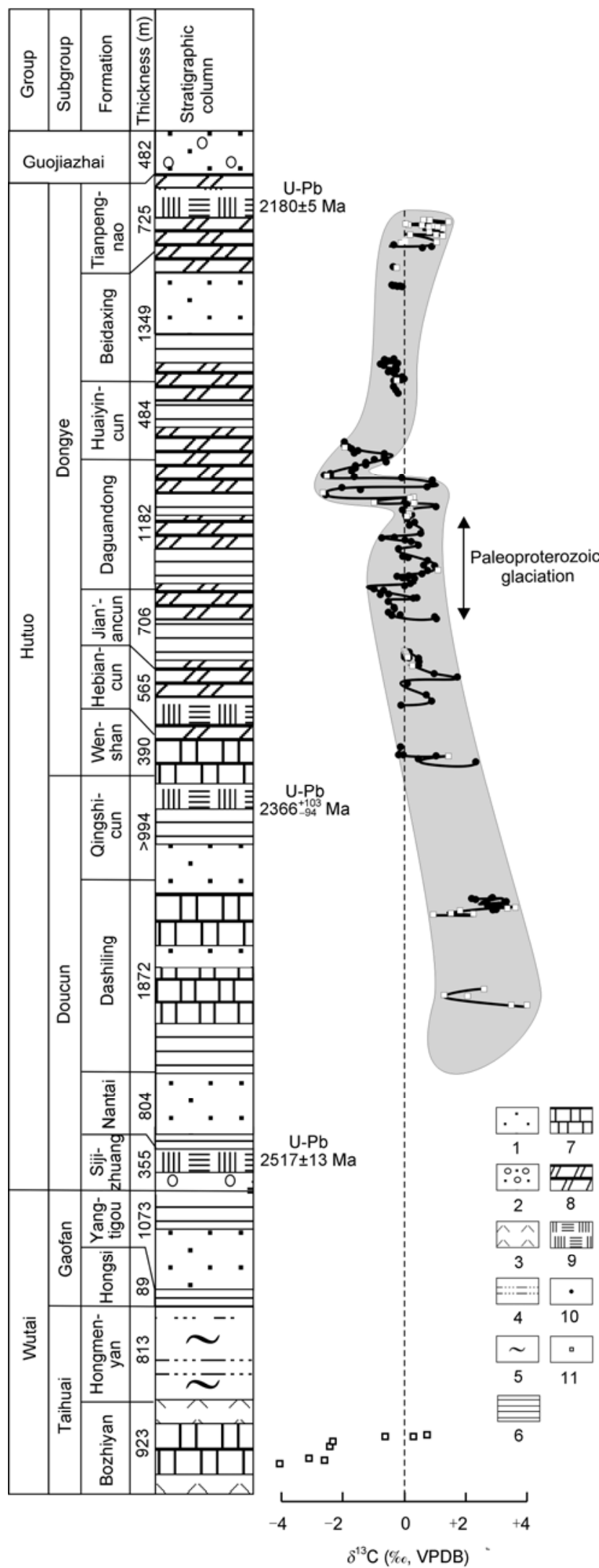

Figure $3 \delta^{13} \mathrm{C}$ profile of the Paleoproterozoic Hutuo Group (zircon ages from Wu et al. [12,13] and Wilde et al. [16]). 1, Quartzite; 2, metacong lomerate; 3, breccia; 4, schist; 5 , greenschist; 6 , shale; 7, marble; 8 , dolomite; 9 , metavolcanics; 10 , samples with $\delta^{18} \mathrm{O}>-10 \%$, 11 , samples with $\delta^{18} \mathrm{O}<-10 \%$. 
growth of stromatolites may also have helped the drawdown of greenhouse gas, an important trigger for global glaciation. A global cold climate depressed oceanic primary productivity and burial of organic matter [22], and accordingly resulted in the negative carbon isotope excursion seen in marine carbonates of this time period. A negative carbon isotopic excursion occurs from the Wenshan Formation upward $\left(\delta^{13} \mathrm{C}_{\text {carb }}\right.$ values shift from $2.0 \%$ to $-1.2 \%$ ), and $\delta^{13} \mathrm{C}_{\text {carb }}$ values fluctuate around $0 \%$ from the Jian'ancun Formation to the middle of the Daguandong Formation.

During the glaciation and transition to an interglacial period, massive release of $\mathrm{CO}_{2}$ from oceanic ridges [23] and methane release from shelf and slope settings [22] into at mosphere-ocean system finally triggered the termination of the glaciation. Organic matter in the deep oceans was oxidized via bacterial sulfate reduction to ${ }^{13} \mathrm{C}$-depleted $\mathrm{HCO}_{3}^{-}$, possibly driven by post-glacial invigoration of deep circulation. The ${ }^{13} \mathrm{C}$-depleted, dissolved inorganic carbon was incorporated into the surface ocean, contributing to the negative $\delta^{13} \mathrm{C}_{\text {carb }}$ values recorded in the micritic carbonates [22]. The pronounced negative carbon isotope excursion at the boundary of the Daguandong and Huaiyincun formations ( 2.35 to $2.2 \mathrm{Ga})\left(\delta^{13} \mathrm{C}_{\text {carb }}\right.$ values shift from $1.4 \%$ o to $-3.3 \%$ ) is here considered to record this process. The negative carbon isotope shift resembles that of cap carbonates in the aftermath of the Neoproterozoic glaciation [22]. Thus, the Paleoproterozoic global glaciation occurred right before this negative excursion.

Negative $\delta^{13} \mathrm{C}_{\text {carb }}$ values range from $-3 \%$ to $-1 \%$ at the boundary of the Daguandong and Huaiyincun formations, which is consistent with previously reported data by Zhong and $\mathrm{Ma}$ [8] and the data from the Huronian Supergroup $\left(\delta^{13} \mathrm{C}_{\text {carb }}-1.3 \pm 0.5 \%\right.$ o $)$ [24]. The present data show less pronounced negative values than those from carbonate cements of the Makganyene Formation diamictite in South Africa ( $\delta{ }^{13} \mathrm{C}_{\text {carb }}$ values: $-15 \%$ o to $-3 \%$ ) [25]. Although no diamictite occurs in the Wutai Mountain area, North China, the present study suggests that the negative carbon isotope excursion at the boundary of the Jian'ancun and Daguandong formations, Hutuo Group, may be a response to the Paleoproterozic worldwide glaciation.

A later positive carbon isotope shift (2.2 to $2.06 \mathrm{Ga}$ ) may be related to post-glacial warm conditions and the breakup of the supercontinent Kenorland [26]. A post-glacial greenhouse condition would have been favorable to planktonic blooms, resulting in increase of oceanic primary productivity and burial of organic matter. The breakup of the supercontinent Kenorland at 2.1 to $2.0 \mathrm{Ga}$ [27] produced many epicontinental shallow sea environments, which provided more living space for organisms. As discussed above, the increase of oceanic primary productivity and burial rate of organic matter may have resulted in the positive shift of carbonate carbon isotope values, i.e. the positive shift of $\delta{ }^{13} \mathrm{C}_{\text {carb }}$ values from the Beidaxing to Tianpengnao for- mations (from $-1.2 \%$ to $1.4 \%$ ).

We thank Tian Yongqing, Meng Fanwei, Xiang Ke, and Song Weiming for field assistance, and Chen Xiaoming for isotopic analysis. This work was supported by the Knowledge Innovation Program of the Chinese Academy of Sciences (KZCX2-YW-153), the National Natural Science Foundation of China (40625006) and the Fundamental Research Funds for the Central Universities (2011QNA04).

1 Pavlov A A, Kasting J F. Mass-independet fractionation of sulfer isotope in Archean sediments: Strong evidence for an anoxic Archean atmosphere. Astrobiology, 2002, 2: 27-41

2 Kirschvink J L, Gaidos E J, Bertani L E. Paleoproterozoic snowball Earth: Extreme climatic and geochemical global change and its biological consequences. Proc Natl Acad Sci USA, 2000, 97: 1400-1405

3 Evans D A, Beukes N J, Kirschvink J L. Low-latitude glaciation in the Palaeoproterozoic era. Nature, 1997, 386: 262-266

4 Kopp R E, Kirschvink J L, Hilburn I A, et al. The paleoproterozoic snowball Earth: A climate disaster triggered by the evolution of oxygenic photosyntheis. Proc Natl Acad Sci USA, 2005, 102: 11131-11136

5 Barley M E, Bekker A, Krapez B. Late Archean to Early Paleoproterozoic global tectonics, environmental change and the rise of atmospheric oxygen. Earth Planet Sci Lett, 2005, 238: 156-171

6 Hayes J M. Global methanotrophy at the Archean-Proterozoic transition. In: Bengston S, ed. Proc Nobel Symp, Early Life on Earth, Vol. 84, New York: Columbia University Press, 1994. 220-236

7 Pavlov A A, Kasting J F, Brown L L. Greenhouse warming by $\mathrm{CH}_{4}$ in the atmosphere of early Earth. J Geophys Res, 2000, 105: 11981-11990

8 Zhong H, Ma Y S. Discovery of early proterozoic carbon isotope shifts (in Chinese). Acta Geol Sin, 1995, 69: 185-190

9 Zhong H, Ma Y S. Oxygen isotope geochemistry of Early Proterozoic Hutuo Group dolomites, Wutaishan Mountain area (in Chinese). Geochimica, 1995, 24(suppl): 83-91

10 Polat A, Kusky T, Li J, et al. Geochemistry of Neoarchean (ca. 2.55-2.50 Ga) volcanic and ophiolitic rocks in the Wutaishan greenstone belt, central orogenic belt, North China craton: Implications for geodynamic setting and continental growth. GSA Bull, 2005, 117: 1387-1399

11 Bai J. Precambrian Geology of Wutai Mountain Area (in Chinese). Tianjing: Science and Technology Press, 1986. 1-435

12 Wu J S, Liu D Y, Geng Y S. Compositive research report ahout Hutuo Group-chronostratigraphic framework and sequence of important geological events (in Chinese). In: Compilation of Achievements in Dividing Stages of Major Faults in China. Beijing: Geological Publishing House, 2005

13 Wu J S, Geng Y S, Jin L G. The zircon U-Pb age of metamorphosed basic volcanic lavas from the Hutuo Group in the Wutai Mountain area, Shanxi Province (in Chinese). Geol Rev, 1985, 32: 178-184

14 Wang R Z, Yan Y Y, Li H M, et al. The early precambrian chronotectonic framework of the Wutaishan area, China. 30th IGC Abstract, 1996, 2: 579

15 Wang R Z, Yan Y Y, Li H M, et al. Geochronology study on the Early Precambrian metamorphic rocks in the Wutaishan area, Shanxi. Acta Geosci Sin, 1996, 151-157

16 Wilde S A, Zhao G, Wang K, et al. New evidence for jig- saw puzzle of Paleoproterozoic North China Craton: Zircon SHRIMP U-Pb age of Hutuo Group of Wutai Mountain (in Chinese). Chinese Sci Bull, 2003, 48: 2180-2186

17 Zhang Y J. Genetic conditions of paleoweathering crust of Qingshicun formation, Hutuo Group (in Chinese). Shanxi Geol, 1990, 5: 260-266

18 Zheng Y F, Chen J F. Stable Isotope Geochemistry (in Chinese). Beijing: Science Press, 2000

19 Kaufman A J, Knoll A H. Neoproterozoic variations in the C-isotope composition of seawater: Stratigraphic and biogeochemical implica- 
tions. Precambrian Res, 1995, 73: 27-49

20 Bekker A, Sial A N, Karhu J A, et al. Chemostratigraphy of carbonates from the Minas Supergroup, Quardilatero Ferrifero (iron quardrangle), stratigrapgic record of early proterozoic atmospheric, biogeochemical and climatic change. Am J Sci, 2003, 303: 865-904

21 Larson R L. Geological consequences of superplumes. Geology, 1991, 19: 963-966

22 Kaufman A J, Knoll A H, Narbonne G M. Isotope, ice ages, and terminal Proterozoic earth history. Proc Natl Acad Sci USA, 1997, 94: 6600-6605

23 Chu X L. "Snowball Earth" during the Neoproterozoic (in Chinese).
Bull Mineral Petrol Geochem, 2004, 23: 233-238

24 Karhu J A, Holland H D. Carbon isotopes and the rise of atmospheric oxygen. Geology, 1996, 24: 867-870

25 Polteau S, Moore J M, Tsikos H. The geology and geochemistry of the Palaeoproterozoic Makganyene diamictite. Precambrian Res, 2006, 148: 257-274

26 Bekker A, Karhu J A, Kaufman A J. Carbon isotope record for the onset of Lomagundi carbon isotope excursion in the Great Lake area, North America. Precambrian Res, 2006, 148: 145-180

27 Aspler L B, Chiarenzelli J R. Two Neoarchean supercontinents? Evidence from the Paleoproterozoic. Sediment Geol, 1998, 120: 75-104

Open Access This article is distributed under the terms of the Creative Commons Attribution License which permits any use, distribution, and reproduction in any medium, provided the original author(s) and source are credited. 